\title{
Improving Unconstrained Iris Recognition Performance via Domain Adaptation Metric Learning Method
}

\author{
Yan Fei ${ }^{1}$, Zhou Changiiu ${ }^{1,3}$, and Tian Yantao ${ }^{1,2 *}$ \\ 1. College of Communication Engineering, Jilin University, \\ Changchun 130025, China \\ 2. Key Laboratory of Bionic Engineering of Ministry of Education, Jilin \\ University, Changchun 130025, China \\ 3. Advanced Robotics and Intelligent Control Centre, Singapore Polytechnic, \\ Singapore \\ yanfei@sina.cn (Yan Fei) \\ tianyt@jlu.edu.cn (Tian Yantao)
}

\begin{abstract}
To improve unconstrained iris recognition system performance in different environments, a performance improvement method of unconstrained iris recognition based on domain adaptation metric learning is proposed. A kernel matrix is calculated as the solution of domain adaptation metric learning. The known Hamming distance computing by intra-class and inter-class is used as the optimization learning constraints in the process of iris recognition. An optimal Mahalanobis matrix is computed for certain cross-environment system, then distance between two iris samples is redefined. The experimental results indicate that the proposed method can increase the accuracy of the unconstrained iris recognition in different circumstances, improving the classification ability of iris recognition system.
\end{abstract}

Keywords: Iris recognition; Metric learning; Similarity measure; Pattern recognition; Biometrics recognition

\section{Introduction}

With the increasing understanding of iris anatomy and development of computer technology, iris recognition technology is widespread in biometric technology research and daily life. Uniqueness of iris texture information is the basis for iris recognition, and modern computer technology makes iris recognition realized rapidly and accurately. Daugman first proposed an automatical iris recognition system in 1993[1]. According to Daugman's thoughts, many researchers have continued with the study on iris segmentation, feature extraction [2-4] and iris comparator [5-8] in subsequent research. Methods mentioned above require highquality iris images for proper functioning, so high precision hardware deployment and strict coordination with users are required. However, with extended use strategy of iris recognition system, less constrained or unconstrained iris recognition systems are becoming more and more necessary $[9,10]$. An unconstrained iris recognition system can relieve environmental restrictions and human cooperation restrictions $[11-14]{ }^{1}$

There are for two ways to improve recognition accuracy of unconstrained iris recognition system. One is the improvement of the performance of image acquisition device, as we know, high-quality iris images can be captured by a

Tian Yantao is the corresponding author. 
sophisticated camera $[15,16]$, and then traditional iris recognition algorithms can be used in the system like normal. But high-performance cameras are generally expensive. It restricts the use of iris recognition systems. On the other hand, we can employ the optimization algorithms to optimize the iris recognition system. Using machine learning methods to optimize the unconstrained iris recognition system can improve recognition accuracy of the system [6, 14, 17-19]. Considering the case of the same system works in different environments, probability distribution of samples will change because distance transformation and illumination change. Different probability distributions will make classification performance drop significantly. Domain adaptation metric learning (DAML) method can resolve the distribution difference between source and target domain data [20-23]. In the unconstrained iris recognition system, training samples distribution and test samples distribution are altered because the environment changes. Therefore, DAML method is utilized to improve recognition accuracy of unconstrained iris recognition system.

The rest of the paper is organized as follows. Section 2 introduces the unconstrained iris recognition system and similarity measure. Section 3 describes the framework for DAML, then solves the DAML under the constraints. The DAML method is used to optimize the feature matching algorithm in Section 4. Experimental setting details and results are shown in Section 5.Finally, the conclusion will be described in section 6 .

\section{Unconstrained Iris Recognition and Similarity Measure}

\subsection{The Unconstrained Iris Recognition System}

An iris recognition system makes up of hardware and software. The hardware includes one image capture device and information processing equipment. Software mainly contains image acquisition algorithm, image preprocessing algorithm, iris segmentation algorithm, feature extraction algorithm and feature matching algorithm. Unconstrained iris recognition system generally follows this classical composition [24-26], Figure 1 illustrates the composition of the iris recognition system. We use symbol * to mark the possible differences between the common system and the unconstrained system.

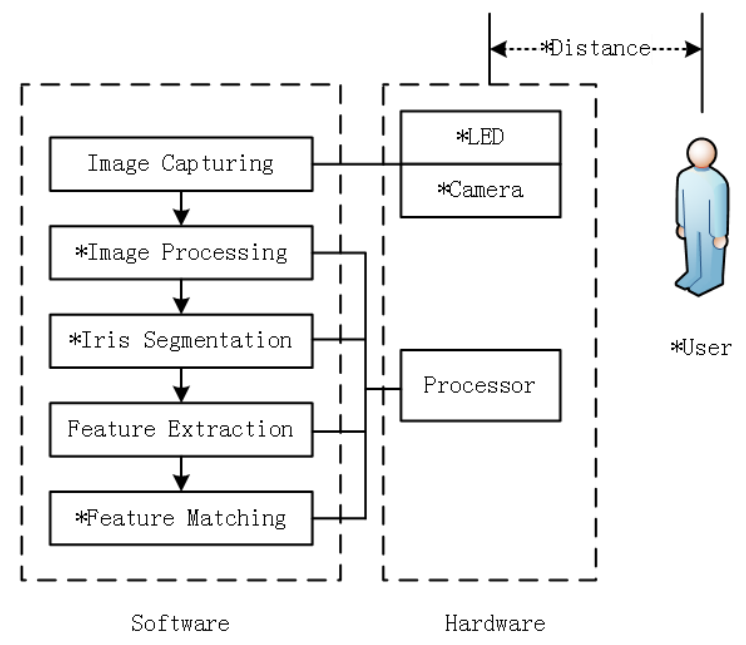

Figure 1. Composition of Iris Recognition System

The iris recognition framework is shown as Figure 1. The possible differences between the common system and unconstrained system are listed in Table 1. 
Table 1. Differences between Common and Unconstrained Iris Recognition

\begin{tabular}{lll}
\hline & Common & Unconstrained \\
\hline User behavior & Strictly cooperate & Less cooperate \\
Working distance & Close(<0.5 meter) & Long(>1 meter) \\
Artificial lighting & Near-infrared LED & None/Visible light \\
Camera performance & Ordinary & High \\
Image Processing & Gray image & Gray/Color image \\
Iris Segmentation & Regular shape & Occlusion culling \\
Feature Matching & Direct & Optimized \\
\hline
\end{tabular}

According to the comparison in Table 1, we can illustrate the unconstrained iris recognition system in more detail. Under the common circumstances, close distance and near-infrared illumination form a stable work environment. In comparison, an unconstrained iris recognition system can work at longer distance, and it does not oblige user to cooperate strictly. In the unconstrained iris recognition system, environmental constraints and user constraints are reduced obviously, so we call it "unconstrained". In order to obtain high-quality iris images, a higher performance camera is often used in the system. In the algorithms, it should take such corresponding changes in image processing, iris segmentation, and feature matching for the unconstrained data.

\subsection{Similarity Measure for Iris Recognition}

When the unconstrained iris recognition system works, environmental changes are frequently encountered. Environmental changes mainly refer to changes in the distance and lighting conditions. Our goal is to improve the recognition accuracy in different environments. That is to say, we want the system to adapt cross-environment situation.

It can simplify the question to the simplest case. Considering the unconstrained iris recognition system only deploys in two environments. It can denote different environments as environment $S_{1}$ and environment $S_{2}$. In environment $S_{1}$, it can obtain a set of iris samples $X_{1}=\left\{\left(x_{a}, y_{c}\right)\right\}$, and $a=1, \mathrm{~L}, m$. In environment $\mathrm{S}_{2}$, it can obtain a set of iris samples $X_{2}=\left\{\left(x_{b}, y_{c}\right)\right\}$, and $b=1, \mathrm{~L}, n$. In the sets, $y_{c}$ is the class label of iris samples. If the total number of categories is $\mathrm{N}$, it can know $c=1, \mathrm{~L}, \mathrm{~N}$. We want the iris recognition system to work properly with greater accuracy on samples set $X=X_{1}+X_{2}$, so DAML method is effective to this target.

In the DAML method, a similarity measure between two iris samples is essential. Establishing an iris features comparator, calculating the distance between the two iris samples, and comparing the distance with the fixed threshold are the major steps of iris feature matching algorithm. There are several frequently-used comparators for iris recognition, such as Hamming distance method [1], Context-based method [7], and Ko's method [8]. Hamming distance approach is used universally and it can bring about good results when utilizing Hamming distance as a comparator. So we choose the Hamming distance method to be the feature matching comparator in the following sections.

After iris segmentation and feature extraction, it can get a set of binary sequences as the sample feature. An iris feature $x$ is composed of two parts, code part $c$ and mask part $m$, that is $x=\left[\begin{array}{ll}c & m\end{array}\right]^{T}$, where the bits in the mask part mark the corresponding code bits valid or not. Let the length of $c$ and $m$ be $L$, then the sample is $x \in\{0,1\}^{2 L}$. The normalized Hamming distance $H\left(x_{i}, x_{j}\right)$ between iris sample $x_{i}$ and $x_{j}$ is defined as Formula (1) $[5,6]$. 


$$
H\left(x_{i}, x_{j}\right)=\frac{\sum_{l=1}^{L}\left\{m_{i}(l) \wedge m_{j}(l) \wedge\left(c_{i}(l) \oplus c_{j}(l)\right)\right\}}{\sum_{l=1}^{L}\left\{m_{i}(l) \wedge m_{j}(l)\right\}}
$$

where $l$ represents the $l$ th bit in $c$ and $m$. Symbol " $\wedge$ " is the logical operator AND. Symbol " $\oplus$ " is the logical operator XOR. The smaller $H\left(x_{i}, x_{j}\right)$ is, the more similarity between $x_{i}$ and $x_{j}$. Under ideal conditions, when $x_{i}=x_{j}, H\left(x_{i}, x_{j}\right)=0$.

\section{DAML Method and Novel Resolving Scheme}

\subsection{Framework of DAML Method}

The core idea of DAML method is to find a more suitable feature mapping to build a new feature space, in which the deviation of the two original different domains will be reduced to the minimum as possible. And then the corresponding learning model will be established in the new feature space [21,22].

Authors give a general description of the DAML problem in [21]. The DAML method usually maps the data relationship into the Reproducing Kernel Hilbert Space (RKHS) [27], then the source domain and target domain can be made into similar distribution by minimizing the maximum deviation. Through this method, the probability distribution problem in two different domains can be solved.

Let transformation $\phi: \mathrm{X} \rightarrow \mathrm{R}^{d}$ denote the function that maps the sample to RKHS. The inner product $\left\langle\phi\left(x_{i}\right), \phi\left(x_{j}\right)\right\rangle$ can be represented as

$k\left(x_{i}, x_{j}\right)=\left\langle\phi\left(x_{i}\right), \phi\left(x_{j}\right)\right\rangle=\phi\left(x_{i}\right)^{T} \phi\left(x_{j}\right)$

where $k\left(x_{i}, x_{j}\right)$ is also known as a kernel function. If the total number of samples is $n$, and the element in a matrix $K$ is $K_{i j}=k\left(x_{i}, x_{j}\right), K$ is known as the kernel matrix.

Let $X_{s}=\left\{\left(x_{i}, y_{i}\right), i=1, \mathrm{~L}, m\right\}$ denote a data set that subject to distribution $Q$, and $x_{i} \in \mathrm{X}$ is the $i$ th sample, $y_{i} \in \mathrm{Y}$ is the corresponding class label. Meanwhile, $X_{t}=\left\{\left(x_{i}, y_{i}\right), i=m+1, \mathrm{~L}, m+n\right\}$ is a data set that subjects to distribution $P$, where $P \neq Q$. The distance between $x_{i}$ and $x_{j}$ in the kernel space is defined as

$D\left(x_{i}, x_{j}\right)=\left\|\phi\left(x_{i}\right)-\phi\left(x_{j}\right)\right\|$

In the particular circumstances, if $\mathrm{X}=\mathrm{R}^{D}$, it can get $\phi\left(x_{i}\right)=L x_{i}$, where $L \in \mathrm{R}^{d \times D}$. Formula (3) can be rewritten as

$D\left(x_{i}, x_{j}\right)=\sqrt{\left(x_{i}-x_{j}\right)^{T} A\left(x_{i}-x_{j}\right)}$

Squaring both sides of Formula (4), it can get a new distance measure in RKHS as

$D_{e}\left(x_{i}, x_{j}\right)=D\left(x_{i}, x_{j}\right)^{2}=\left(x_{i}-x_{j}\right)^{T} A\left(x_{i}-x_{j}\right)$

where $A=L^{T} L$ is a symmetric positive semidefinite matrix, it is also known as Mahalanobis distance matrix. Mahalanobis distance can be counted as the Euclidean distance after scale variation and rotation transformation on the feature space. The purpose of DAML is to obtain the optimized transformation matrix $A$ by making use of features relationship of samples in $X_{s}$ and $X_{t}$. 


\subsection{Constraints for Optimal Matrix Learning}

Considering that there is a set of constraints to judge whether two samples belong to the same class. If the distance between samples is less than a given upper limit, then the two samples belong to the same class. In contrast, if the distance between samples is more than a given lower limit, then the two samples are different. Let $X=\left\{x_{1}, \mathrm{~L}, x_{n}\right\}$ denote a sample set in space $R^{d}$, the distance between $x_{i}$ and $x_{j}$ is $D_{e}\left(x_{i}, x_{j}\right)$, and $x_{i}, x_{j} \in X$. The relationship between $x_{i}$ and $x_{j}$ can be classified into two types, same class or not. Let $S$ denote the same class set, $D$ denotes the different class set, then there must be an upper limit $u$ and lower limit $l$ for $\mathrm{c} 1 . D_{e}\left(x_{i}, x_{j}\right) \leq u$, if $\left(x_{i}, x_{j}\right) \in S$, and $\mathrm{c} 2 . D_{e}\left(x_{i}, x_{j}\right) \geq l$, if $\left(x_{i}, x_{j}\right) \in D$.

The constraint $\mathrm{c} 1$ and $\mathrm{c} 2$ for DAML must be followed in the learning process, and iris recognition across different environments also obeys above constraints.

$D_{e}\left(x_{i}, x_{j}\right)$ in Formula (3) is the distance measure between samples in set $X_{s}$ and $X_{t}$. It is assumed that there is a valid distance measure $D_{x}$ for set $X_{s}$, and $D_{x}$ is also effective to $X_{t}$. The distribution of $X_{s}$ and $X_{t}$ is different, so $D_{x}$ cannot meet the essential demand of distance measure when the distribution of $X_{s}$ and $X_{t}$ disparity is oversized. To solve this problem, we can optimize the transform matrix $A$, then the samples in $X_{s}+X_{t}$ will be remapped to obtain the new distance measure $D_{e}$. The process of learning optimization is to find the optimal matrix $A$. Then it can make the difference between $D_{e}$ and $D_{x}$ to reach minimum under constrain conditions. Thus the DAML can be expressed as

$$
A^{*}=\underset{\phi: \mathrm{X} \rightarrow R^{M}}{\arg \min } \sum_{x_{i}, x_{j} \in\left(X_{s}+X_{t}\right)} D_{a}\left(D_{e}\left(x_{i}, x_{j}\right), D_{x}\left(x_{i}, x_{j}\right)\right)
$$

subject to $D_{e}\left(x_{i}, x_{j}\right) \leq u$, if $\left(x_{i}, x_{j}\right) \in S$, and $D_{e}\left(x_{i}, x_{j}\right) \geq l$, if $\left(x_{i}, x_{j}\right) \in D$, where $A^{*}$ is the optimal Mahalanobis distance matrix, $D_{a}$ is a suitable distance measure which can be defined as $D_{a}(p, q)=\|p-q\|$. Substituting $A^{*}$ for Formula (5), the optimal distance measure between $x_{i}$ and $x_{j}$ can be represented as

$D_{e}\left(x_{i}, x_{j}\right)=\left(x_{i}-x_{j}\right)^{T} A^{*}\left(x_{i}-x_{j}\right)$.

\subsection{Kernel Solution of Optimal Mahalanobis Distance Matrix}

In section 3.1, we have mentioned a special case. In the transformation $\phi: \mathrm{X} \rightarrow \mathrm{R}^{d}$, if $\mathrm{X}=\mathrm{R}^{D}$, it can know that $f\left(x_{i}\right)=L x_{i}$, where $L \in \mathrm{R}^{d \times D}$. By substituting Formula (3), we can rewrite the kernel function as

$k\left(x_{i}, x_{j}\right)=\phi\left(x_{i}\right)^{T} \phi\left(x_{j}\right)=\left(L x_{i}\right)^{T}\left(L x_{j}\right)=x_{i}^{T}\left(L^{T} L\right) x_{j}=x_{i}^{T} A x_{j}$

Given a sample set $X=\left[\begin{array}{llll}x_{1} & x_{2} & \mathrm{~L} & x_{n}\end{array}\right]$, the relationship between the kernel matrix $K$ and Mahalanobis distance matrix $A$ is

$K=X^{T} A X$

Formula (8) shows the relationship between the kernel matrix $K$ and Mahalanobis distance matrix $A$, so the problem of computing the optimal Mahalanobis distance matrix $A^{*}$ can be converted into computing the optimal kernel matrix $K^{*}$. By computing $A^{*}$, we can solve Formula (8) as

$A^{*}=\left(X K^{*-1} X^{T}\right)^{-1}$ 
The relationship between new distance measure $D_{e}$ and the kernel matrix is

$$
\begin{aligned}
D_{e}\left(x_{i}, x_{j}\right) & =\left\|\phi\left(x_{i}\right)-\phi\left(x_{j}\right)\right\|^{2} \\
& =\phi\left(x_{i}\right)^{T} \phi\left(x_{i}\right)+\phi\left(x_{j}\right)^{T} \phi\left(x_{j}\right)-2 \phi\left(x_{i}\right) \phi\left(x_{j}\right) \\
& =K_{i i}+K_{j j}-2 K_{i j}
\end{aligned}
$$

Therefore, the Formula (6) can be rewritten in a kernel matrix optimization problem as

$$
K^{*}=\underset{\phi: \mathrm{X} \rightarrow R^{M}}{\arg \min } \sum_{x_{i}, x_{j} \in\left(X_{s}+X_{t}\right)} D_{a}\left(K_{i i}+K_{j j}-2 K_{i j}, D_{x}\left(x_{i}, x_{j}\right)\right)
$$

subject to $K_{i i}+K_{j j}-2 K_{i j} \leq u$, if $\left(x_{i}, x_{j}\right) \in S$, and $K_{i i}+K_{j j}-2 K_{i j} \geq l$, if $\left(x_{i}, x_{j}\right) \in D$.

A method is proposed in [6] for calculating the optimal kernel matrix $K^{*}$. The problem of optimizing the kernel matrix can be expressed as

$K^{*}=\underset{K \in P S D}{\arg \min } D_{l d}\left(K, K_{0}\right)$

where $K_{0}$ is a known kernel, and $D_{l d}$ is the divergence of LogDet Matrix. The value of $K_{t+1}$ in the $(t+1)$ th iteration can be calculated as

$K_{t+1}=K_{t}+\beta_{t+1} K_{t} e_{t_{i}} e_{t_{j}}^{T} K_{t}$

Let $C=\{(i, j)\}$ be the sequence numbers set of all constraints, where $(i, j)$ corresponds to a constrain imposed between training samples $x_{i}$ and $x_{j}$. The number of constraints is $N$. Let the constraint chosen after the $t$ th iteration be formed using the $t_{i}$ th and the $t_{j}$ th data samples, then $\left(t_{i}, t_{j}\right) \in C . e_{t_{i}}$ and $e_{t_{j}}$ are the position vector of $t_{i}$ and $t_{j}$. Elements in $e_{t_{i}}$ and $e_{t_{j}}$ are 1 corresponding to the position of $t_{i}$ and $t_{j}$ other positions are 0 . When $t=0, K_{t}=K_{0} \cdot \beta_{t+1}$ is mapping parameters which can be solved according to Algorithm 1.

\section{Algorithm 1. Algorithm to Compute $\beta+1$}

Input: Kernel Matrix at the $t$ th iteration $K_{t}$, Constraints set $C$, upper limit $u$, lower limit $l$, relaxation parameter $\gamma$.

Output: $\beta_{t+1}$.

\section{Initialization:}

1. if $t=0$,

$\lambda_{i j}^{(t)}=0, \forall(i, j) \in C$.

$\varphi_{i j}^{(t)}=u$, if $y_{t_{i}}=y_{t_{j}}, \varphi_{i j}^{(t)}=l$, otherwise.

2. $\delta=1$ if $y_{t_{i}}=y_{t_{i}}, \delta=-1$, if otherwise.

\section{Parameter Update:}

$$
\begin{aligned}
& p=K_{t_{t} t_{i}}^{(t)}+K_{t_{j} t_{j}}^{(t)}-2 K_{t_{i} t_{j}}^{(t)} . \\
& \alpha=\min \left(\lambda_{t_{i} t_{j}}^{(t)}, \frac{\delta}{2}\left(\frac{1}{p}-\frac{\gamma}{\varphi_{t_{t}}^{(t)}}\right)\right) . \\
& \beta_{t+1}=\frac{\delta \alpha}{(1-\delta \alpha p)} . \\
& \lambda_{t_{i} t_{j}}^{(t+1)}=\lambda_{t_{t} t_{j}}^{(t)}-\alpha . \\
& \varphi_{t_{t} t_{j}}^{(t+1)}=\frac{\gamma \varphi_{t_{t}}^{(t)}}{\gamma+\delta \alpha \varphi_{t_{i}}^{(t)}} .
\end{aligned}
$$




$$
\begin{aligned}
& \lambda_{i j}^{(t+1)}=\lambda_{i j}^{(t+1)}, \forall(i, j) \in C,(i, j) \neq\left(t_{i}, t_{j}\right) . \\
& \varphi_{i j}^{(t+1)}=\varphi_{i j}^{(t+1)}, \forall(i, j) \in C,(i, j) \neq\left(t_{i}, t_{j}\right) .
\end{aligned}
$$

Then the optimal kernel matrix $K^{*}$ can be computed as

$K^{*}=K_{0}+\sum_{t=1}^{N} \beta_{t} K_{t-1} e_{t_{i}} e_{t_{j}}^{T} K_{t-1}$

The optimal Mahalanobis distance matrix $A^{*}$ is

$$
A^{*}=\left(X\left(K_{0}+\sum_{t=1}^{N} \beta_{t} K_{t-1} e_{t_{i}} e_{t_{j}}^{T} K_{t-1}\right)^{-1} X^{T}\right)^{-1}
$$

\section{Optimization Algorithm Based on DAML Method}

\subsection{Optimal Target in Unconstrained Iris Recognition}

The cross-environment condition in unconstrained iris recognition is described above, then we give a derivation of DAML method in section 3. In order to explain the suitability of DAML method, the problems of adapting cross-environment in unconstrained iris recognition can be restated.

An unconstrained iris recognition system runs in environment $S_{1}$ and environment $S_{2}$. It can gain an iris samples set $X_{1}=\left\{\left(x_{a}, y_{c}\right)\right\}$ in environment $\mathrm{S}_{1}$, where $a=1, \mathrm{~L}, m$ is an ordinal number, $y_{c}$ is the corresponding class label. The set $X_{1}$ subjects to the distribution $P$. In environment $\mathrm{S}_{2}$, it can gain an iris samples set $X_{2}=\left\{\left(x_{b}, y_{c}\right)\right\}$, where $b=1, \mathrm{~L}, n$. The set $X_{2}$ subjects to the distribution $Q$. We define that distribution $\mathrm{S}_{1}$ and distribution $\mathrm{S}_{2}$ are different environments, so the distribution $P \neq Q$. Then the problem of the environment adaptability in unconstrained iris recognition is converted into DAML problem.

Hamming distance is a type of effective similarity measure between iris samples in the same distribution. In different environments, iris samples distribution is different, then we can compute the optimal Mahalanobis distance matrix $A^{*}$ by using the known Hamming distance. The new distance measure $D_{e}$ and Hamming distance measure will have minimum distinction under the constraints. Formula (6) can be rewritten as

$$
A^{*}=\underset{\phi: \mathrm{X} \rightarrow R^{M}}{\arg \min } \sum_{x_{i}, x_{j} \in\left(X_{s}+X_{t}\right)} D_{a}\left(D_{e}\left(x_{i}, x_{j}\right), H\left(x_{i}, x_{j}\right)\right)
$$

where $D_{e}$ is defined in Formula (5). $D_{a}$ is a distance between $D_{e}$ and Hamming distance $H$, it can be denoted as $D_{a}\left(D_{e}, H\right)=\left\|D_{e}-H\right\| . A^{*}$ is the optimal Mahalanobis distance matrix subject to the constraints. The problem of solving $A^{*}$ can transform into solving the optimal kernel matrix $K^{*}$. Formula (11) can be written as

$$
K^{*}=\underset{\phi: \mathrm{X} \rightarrow R^{M}}{\arg \min } \sum_{x_{i}, x_{j} \in\left(X_{s}+X_{t}\right)} D_{a}\left(K_{i i}+K_{j j}-2 K_{i j}, H\left(x_{i}, x_{j}\right)\right)
$$

In the process of optimize learning, constraint conditions must be considered. Like the description in section 3.2. The constraints are discussed in two different cases.

Case 1: The iris samples obey the same distribution. If $x_{i}, x_{j} \in X_{1}$ or $x_{i}, x_{j} \in X_{2}$ is satisfied, the constraints are c1. $D_{e}\left(x_{i}, x_{j}\right) \leq u$, if $\left(x_{i}, x_{j}\right) \in S$, and c2. $D_{e}\left(x_{i}, x_{j}\right) \geq l$, if $\left(x_{i}, x_{j}\right) \in D$.

Under the constraints of case 1, it can ensure that the system performance will not reduce in single work environment. 
Case 2: The iris samples obey different distributions. If $x_{i} \in X_{1}, x_{j} \in X_{2}$ or $x_{j} \in X_{1}, x_{i} \in X_{2}$ is satisfied, the constraints are c1. $D_{e}\left(x_{i}, x_{j}\right) \leq u$, if $\left(x_{i}, x_{j}\right) \in S$, and $\mathrm{c} 2$. $D_{e}\left(x_{i}, x_{j}\right) \geq l$, if $\left(x_{i}, x_{j}\right) \in D$.

When working in different environments, the above constraints can aggrandize the similarity of the same class. The distance between two iris samples from the same class should be decreased, and the distance between two samples from different classes should be increased. Therefore, the purpose of optimizing the system performance can be achieved.

Case 1 and case 2 has the same expression form, but they have completely different practical significances. In unconstrained iris recognition, those two cases both exist at the same time, so the precondition can be written as iris samples $x_{i}, x_{j} \in X$, and $X=X_{1}+X_{2}$.

\subsection{Calculating Optimal Matrix for Iris Recognition}

Solving the optimization problem expresses in Formula (17), we can convert it into the optimization problem shown as the Formula (12). The key problem is to find distance measure $K_{0}$ as the similarity matrix. Formula (1) can be expressed as

$H\left(x_{i}, x_{j}\right)=\frac{\eta_{D}\left(x_{i}, x_{j}\right)}{\eta\left(x_{i}, x_{j}\right)}$

where $\eta\left(x_{i}, x_{j}\right)$ denotes the sum of all valid bits of sample $x_{i}$ and $x_{j}$ in feature matching. $\eta_{D}\left(x_{i}, x_{j}\right)$ denotes the sum of disagreement bits in feature matching. Then the sum of agreement bits can be defined as

$\eta_{A}\left(x_{i}, x_{j}\right)=\eta\left(x_{i}, x_{j}\right)-\eta_{D}\left(x_{i}, x_{j}\right)$

Let $H\left(x_{i}, x_{j}\right), \eta\left(x_{i}, x_{j}\right), \eta_{A}\left(x_{i}, x_{j}\right), \eta_{D}\left(x_{i}, x_{j}\right)$ simplify as $H, \eta, \eta_{A}, \eta_{D}$. The Hamming distance can be written as

$H=\frac{\eta_{D}}{\eta}=\frac{2 \eta_{D}+2 \eta_{D}}{4 \eta}=\frac{2\left(\eta-\eta_{A}\right)+2 \eta_{D}}{4 \eta}=\frac{1}{4}+\frac{1}{4}-\frac{\eta_{A}-\eta_{D}}{2 \eta}$

If there is a function

$f(x)=\frac{\left(c(l)-\frac{1}{2}\right) m(l)}{\sqrt{\sum_{l=1}^{L} m(l)}}, \quad l=1, \mathrm{~L}, L$.

It can get another distance measure $F\left(x_{i}, x_{j}\right)$ between $x_{i}$ and $x_{j}$ as

$$
\begin{aligned}
F\left(x_{i}, x_{j}\right) & =f\left(x_{i}\right)^{T} f\left(x_{j}\right) \\
& =\frac{\sum_{l=1}^{L}\left(c_{i}(l)-\frac{1}{2}\right) m_{i}(l)\left(c_{j}(l)-\frac{1}{2}\right) m_{j}(l)}{\sqrt{\sum_{l=1}^{L} m_{i}(l)} \sqrt{\sum_{l=1}^{L} m_{j}(l)}} \\
& =\frac{\sum_{l=1}^{L} m_{i}(l) m_{j}(l)\left(2 c_{i}(l)-1\right)\left(2 c_{j}(l)-1\right)}{4 \sqrt{\sum_{l=1}^{L} m_{i}(l)} \sqrt{\sum_{l=1}^{L} m_{j}(l)}} \\
& =\frac{\eta_{A}-\eta_{D}}{4 \eta}
\end{aligned}
$$

When $x_{i}=x_{j}$, it can get $\eta_{D}=0$ and $\eta=\eta_{A}$. Hence,

$F\left(x_{i}, x_{i}\right)=F\left(x_{j}, x_{j}\right)=\frac{1}{4}$

So we can rewrite Formula (20) as 
$H\left(x_{i}, x_{j}\right)=F\left(x_{i}, x_{i}\right)+F\left(x_{j}, x_{j}\right)-2 F\left(x_{i}, x_{j}\right)$

Formula (23) shows the relationship between $F\left(x_{i}, x_{j}\right)$ and $F\left(x_{i}, x_{j}\right)$. Hamming distance is considered to be an ideal distance measure in the same domain. Contrasting with Formula (10), $F_{i j}=F\left(x_{i}, x_{j}\right)$ can be taken as the known matrix for computing optimal kernel matrix $K^{*}$, so Formula (17) can be rewritten as

$K^{*}=\underset{K \in P S D}{\arg \min } D_{l d}(K, F)$

subject to $K_{i i}+K_{j j}-2 K_{i j} \leq u$, if $\left(x_{i}, x_{j}\right) \in S$, and $K_{i i}+K_{j j}-2 K_{i j} \geq l$, if $\left(x_{i}, x_{j}\right) \in D$.

Constraints generate by all known classification $\left(x_{i}, x_{j}\right)$. Matrix $F$ can compute as

$F_{i j}=\frac{1}{4}-\frac{1}{2} H_{i j}$

where $H_{i j}=H\left(x_{i}, x_{j}\right)$ is Hamming distance between iris sample $x_{i}$ and $x_{j}$.

Let $F$ be the similarity matrix, we can get the solution of Formula (24) by executing Algorithm 1. Finally, the optimal kernel matrix is obtained as

$K^{*}=F+\sum_{t=1}^{N} \beta_{t} K_{t} e_{t_{i}} e_{t_{j}}^{T} K_{t}$,

by substituting into Formula (15), the optimal Mahalanobis distance matrix in unconstrained iris recognition is

$A^{*}=\left(X\left(F+\sum_{t=1}^{N} \beta_{t} K_{t} e_{t_{i}} e_{t_{j}}^{T} K_{t}\right)^{-1} X^{T}\right)^{-1}$

where $X=\left[\begin{array}{llll}x_{1} & x_{2} & \mathrm{~L} & x_{n}\end{array}\right]$ is the training iris samples, it also calls registered samples in iris recognition, and $X=X_{1}+X_{2}$.

\subsection{Iris Feature Matching}

After gaining the optimal Mahalanobis distance matrix $A^{*}$, distance between $x_{i}$ and $x_{j}$ can be calculated by formula (5). According to the requirement of the system, a threshold $\tau$ can be calculated for judging the classification of an unknown sample. The calculation method of $\tau$ is the same as the method of Hamming distance comparator. If the system has a higher security requirement, $\tau$ will set to a smaller value, then the system has a higher false reject rate (FAR). Given a test iris sample $x_{t}$, the distance between $x_{t}$ and the registered samples $X=\left[\begin{array}{llll}x_{1} & x_{2} & \mathrm{~L} & x_{n}\end{array}\right]$ should be calculate by

$D_{e}\left(x_{t}, x_{i}\right)=\left(x_{t}-x_{i}\right)^{T} A^{*}\left(x_{t}-x_{i}\right), \quad i=1, \mathrm{~L}, n$

We can get a distance matrix by formula (27). Let the distance matrix $T_{t i}=D_{e}\left(x_{t}, x_{i}\right)$, it should find the minimum value $T_{t m}$ in matrix $T$. The element $T_{t m}$ is corresponds to the registered sample $x_{m}$. Judgment rules of $x_{t}$ classification are following as

Rule 1: If $T_{t m} \leq \tau$, the test sample $x_{t}$ and registered sample $x_{m}$ is belonging to the same class, that is $y_{t}=y_{m}$.

Rule 2: If $T_{t m}>\tau$, the test sample $x_{t}$ and registered sample $x_{m}$ come from different classes. $x_{t}$ is different from another registered classes. In other words, the class corresponding to $x_{t}$ is not registered in the system. 


\section{Experimental Details and Results}

\subsection{Unconstrained Iris Image Dataset}

Unconstrained iris database ubiris.v2 [28] is used in our experiment. The dataset is an iris database that built by simulating iris image acquisition in less constraint environment. Images in ubiris.v2 are based on RGB space with a resolution of 400 by 300 pixels. Those images are acquired in two different environments named with "S1" and "S2" respectively. The final numbers in the image file name, "I10" and "I13", denote that the images are acquired at five meters distance and four meters distance respectively from the front. The differences among the images are shown in Figure 2. The images shown in Figure 2(a) and Figure 2(b) are acquired in environment S1 at five meters distance and four meters distance. The images shown in Figure 2(c) and Figure 2(d) are images acquired in the environment $\mathrm{S} 2$ at five meters distance and four meters distance.

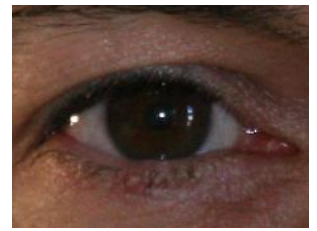

(a) C1_S1_I10

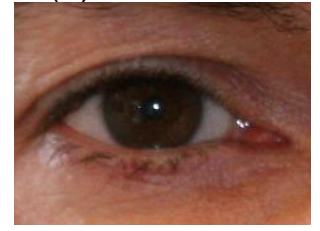

(c) C1_S2_I10
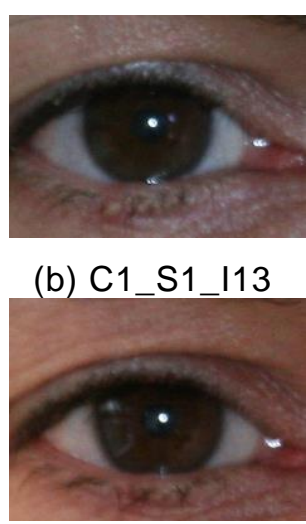

(d) C1_S2_I13

Figure 2. Iris Image Acquired in Unconstrained Environment

\subsection{Segmentation and Feature Extraction}

The main goal of our proposed method is to enhance the accuracy of unconstrained iris recognition system. Thus the focus of the study is not on iris segmentation or feature extraction. Hofbauer et al proposed a segmentation ground truth for some iris databases [29] and provided a partial segmentation mask of ubiris.v2. The segmentation method is used in our experiment. Depending on the experimental need, we complemented some missing data. Follow these facts, a circular pupil was surrounded by iris region, and eyelids were the split boundaries of iris region. We apply Masek's method [30] to iris feature extraction. The 1D Log-Gabor filters are used to extract feature for the rectangular area obtained by the existing segmentation data. Every iris sample will generate a 1280 bits iris feature code.

\subsection{Experimental Settings}

We select one hundred classes of iris in ubiris.v2 for our experiment. All of those samples are acquired at five meters distance and four meters distance. It contains four images in every class of fifty classes. And the four images are acquired in environment S1 and S2. In the remaining fifty classes, it contains two images in every class, which are acquired in environment $\mathrm{S} 1$.

In the iris feature comparison process, there have 350 times for intra-class comparison. Obviously, there will be far more times of inter-class comparison than the intra-class if the inter-class samples are compared one by one between different classes. Thus we limit the comparison times between two samples from different classes. We compare the samples 
from $i$ th class with the samples from $(i+1)$ th class and $(i+2)$ th class. And samples from the ninety-ninth class will be compared with the samples from the hundredth class and the first class, similarly, the samples from the hundredth class will be compared with the samples from the first class and the second class. The total number of inter-class comparison is 1856 times.

The upper limit $u$ and lower limit $l$ in Formula (24) are determined by the distribution of Hamming distance. The obtained intra-class Hamming distance will be sorted in descending order, then select the value at $15 \%$ position of sequence as $u$ value. Meanwhile, the inter-class Hamming distances are sorted in ascending order. We select the value at $15 \%$ position of ascending sequence as $l$ value. In this way, we can obtain that $u=0.4676$ and $l=0.4845$. In DAML method, Hamming distances between iris samples are the training samples for optimal Mahalanobis distance matrix. So it will form 350 constraints that $\left(x_{i}, x_{j}\right) \in S$, and 1856 constraints that $\left(x_{i}, x_{j}\right) \in D$. Relaxation factor in Algorithm 1 is set to $\gamma=0.1$ in the experiment.

\subsection{Experimental results}

To express the performance improvement by the proposed DAML method in unconstrained iris recognition more visually, experimental results are shown as distance distribution between iris samples and the Receiver Operating Characteristic (ROC) curve. The distribution of Hamming distance is shown in Figure 3, where X-axis is the Hamming distance, and Y-axis is the number of distribution. We can calculate that, the mean Hamming distance of intra-class is 0.3584 , and the mean for inter-class is 0.6033 .

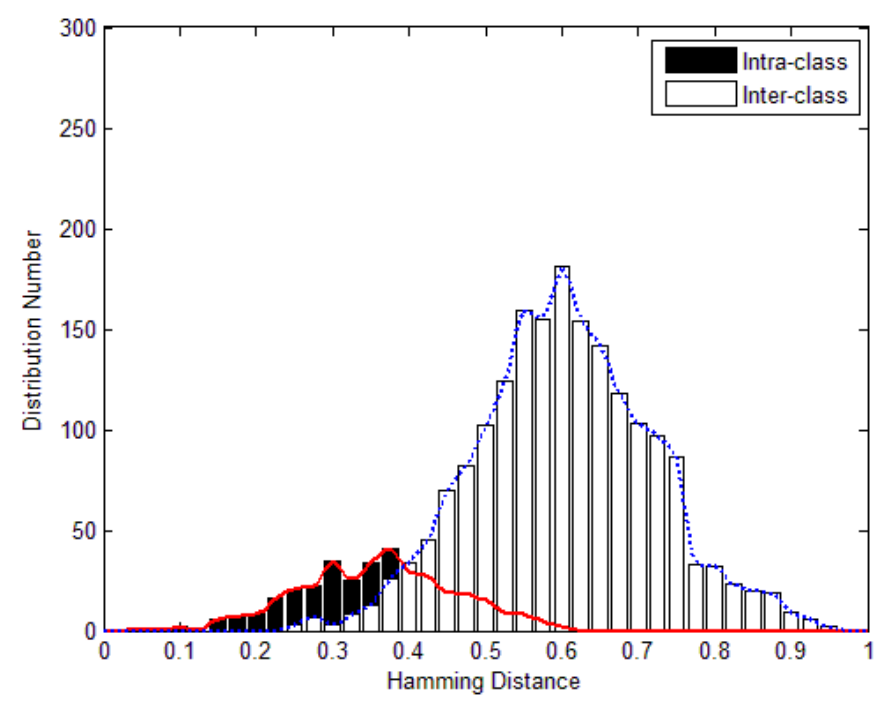

Figure 3. Distribution of Hamming Distance

After using DAML method, the distance between iris samples has been modified. Optimized distance distribution is shown in Figure 4, in which X-axis is the optimized distance, and Y-axis is the corresponding number. After optimizing, the optimized distance mean of intra-class is 0.3520 , and the mean for inter-class is 0.6202 . 


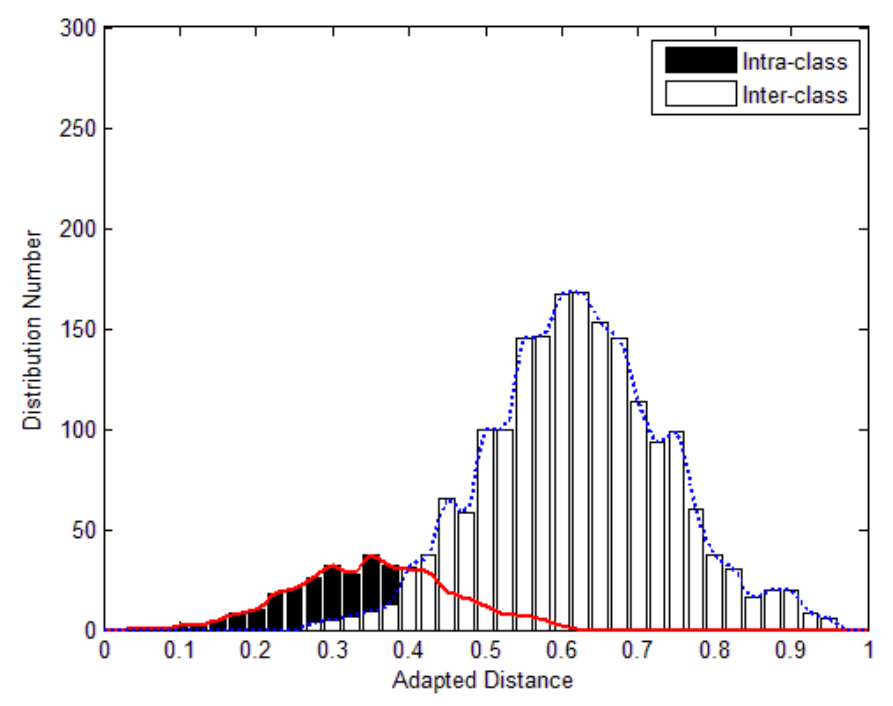

Figure 4. Distribution of Optimal Distance

The ROC curves of Hamming distance measure and optimized measure are shown in Figure 5. The ROC curve is based on False Accept Rate (FAR) as X-axis and True Accept Rate (TAR) as Y-axis, and it is an important performance indicator in biometric information recognition technology. While FAR $=5.00 \%$, the Hamming distance measure is corresponding to TAR $=75.16 \%$, and the optimized distance measure is corresponding to $\mathrm{TAR}=80.33 \%$. While $\mathrm{FAR}=10.00 \%$, the Hamming distance measure is corresponding to $\mathrm{TAR}=83.73 \%$, and the optimized distance measure is corresponding to $\mathrm{TAR}=87.82 \%$.

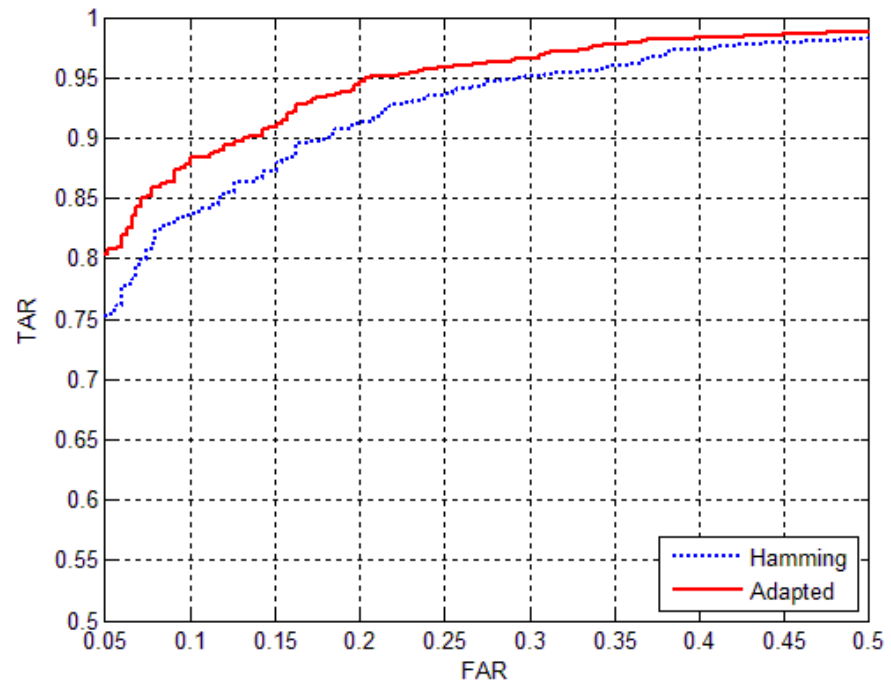

Figure 5. ROC Curve of DAML Optimized

Experimental results above show that, good results cannot be obtained if Hamming distance is directly used as the distance measure. To enhance environment adaptability of unconstrained iris recognition and improve the system performance, we can generate constraints by making use of the existing distance measure, and apply DAML method to optimize the distance measure in iris matching process.

The mean value of intra-class and inter-class is 0.3584 and 0.6033 respectively before optimization. After optimized by using the proposed method, two values change to 0.3520 and 0.6202 respectively. The distance distribution before and after distance measure 
optimization reflects that DAML optimized method makes the intra-class distance smaller, and the inter-class distance larger. Obviously, this change is more conducive to classify unknown iris sample in cross-environment situation. Besides, the ROC curve optimized by DAML method shows that the system performance has been improved after optimization. Calculation of optimal Mahalanobis distance matrix will cost extra time, but the matrix is fixed if the system environment does not change. Thus, the decision time will not increase significantly if we calculate the optimal Mahalanobis distance matrix for different environments in advance.

\section{Conclusion}

Environment adaptability is an important feature for unconstrained iris recognition system. To improve the environment adaptability of iris recognition system, in addition to the influence of hardware performance, we can also optimize the system by using iris recognition results in different environments. In this paper, we introduce the DAML method into iris recognition, using the known distance measures as the optimal constraints, and redefine the distance between iris samples by optimal matrix. The accuracy of iris classification has been improved after using the optimal distance. Thus the performance of the unconstrained iris recognition system is increased.

\section{Acknowledgments}

This paper is supported by the Major Scientific and Technological Achievements Transformation Programs of China (Grant No. 2012-258) and the Jilin University "985 Project” Engineering Bionic Sci. \& Tech. Innovation Platform.

\section{References}

[1] J. G. Daugman, "High confidence visual recognition of persons by a test of statistical independence", IEEE Transactions on Pattern Analysis and Machine Intelligence, vol. 15, no. 11, (1993), pp. 1148-1161.

[2] J. Huang, X. You, Y. Yuan, F. Yang, and L. Lin, "Rotation invariant iris feature extraction using Gaussian Markov random fields with non-separable wavelet", Neurocomputing, vol. 73, no. 4, (2010), pp. 883894.

[3] M. Vatsa, R. Singh, and A. Noore, "Reducing the False Rejection Rate of Iris Recognition Using Textural and Topological”, International Journal of Signal Processing, vol. 2, no. 2, (2005), pp. 66-72.

[4] P. Zhang, L. Zhang, "A Practical and Fast Method of Iris Location", Journal of Harbin University of Science and Technology, vol. 10, no. 5, (2005), pp. 57-59, 63.

[5] J. Daugman, "The importance of being random: statistical principles of iris recognition", Pattern recognition, vol. 36, no. 2, (2003), pp. 279-291.

[6] J. K. Pillai, M. Puertas, and R. Chellappa, "Cross-Sensor Iris Recognition through Kernel Learning", IEEE Transactions on Pattern Analysis and Machine Intelligence, vol. 36, no. 1, (2014), pp. 73-85.

[7] C. Rathgeb, and A. Uhl, "Context-based biometric key generation for iris", IET computer vision, vol. 5, no. 6, (2011), pp. 389-397.

[8] J. G. Ko, Y. H. Gil, J. H. Yoo, and K. I. Chung, "A novel and efficient feature extraction method for iris recognition”, ETRI journal, vol. 29, no. 3, (2007), pp. 399-401.

[9] G. Santos, and E. Hoyle, "A fusion approach to unconstrained iris recognition", Pattern Recognition Letters, vol. 33, no. 8, (2012), pp. 984-990.

[10] K. W. Bowyer, K. P. Hollingsworth, and P. J. Flynn, "A survey of iris biometrics research: 2008-2010", in Handbook of iris recognition, Springer-Verlag London, London, UK, (2013), pp. 15-54.

[11] H. Proença, "Iris recognition: A method to segment visible wavelength iris images acquired on-the-move and at-a-distance", 4th International Symposium on Visual Computing, (2008) December 1-3; Las Vegas, Nevada, USA, pp. 731-742.

[12] K. Y. Shin, G. P. Nam, D. S. Jeong, D. H. Cho, B. J. Kang, K. R. Park, and J. Kim, "New iris recognition method for noisy iris images", Pattern Recognition Letters, vol. 33, no. 8, (2012), pp. 991-999.

[13] H. Proença, "Iris recognition in the visible wavelength", in Handbook of Iris Recognition, SpringerVerlag London, London, UK, (2013), pp. 151-169.

[14] J. Liu, Z. Sun, and T. Tan, "Distance metric learning for recognizing low-resolution iris images", Neurocomputing, vol. 144, (2014), pp. 484-492.

[15] Y. Gong, D. Zhang, P. Shi, and J. Yan, "Optimal wavelength band clustering for multispectral iris recognition", Applied optics, vol. 51, no. 19, (2012), pp. 4275-4284. 
[16] K. Nguyen, C. Fookes, S. Sridharan, and S. Denman, "Quality-driven super-resolution for less constrained iris recognition at a distance and on the move", IEEE Transactions on Information Forensics and Security, vol. 6, no. 4, (2011), pp. 1248-1258.

[17] H. A. Park, and K. R. Park, "Iris recognition based on score level fusion by using SVM", Pattern Recognition Letters, vol. 28, no. 15, (2007), pp. 2019-2028.

[18] Q. Wang, X. Zhang, M. Li, X. Dong, Q. Zhou, and Y. Yin, "Adaboost and multi-orientation 2D Gaborbased noisy iris recognition”, Pattern Recognition Letters, vol. 33, no. 8, (2012), pp. 978-983.

[19] L. Omelina, B. Jansen, M. Oravec, and J. Cornelis, "Feature Extraction for Iris Recognition Based on Optimized Convolution Kernels", the 17th International Conference on Image Analysis and Processing, (2013) September 9-13; Naples, Italy, pp. 141-150.

[20] K. Saenko, B. Kulis, M. Fritz, and T. Darrell, "Adapting visual category models to new domains", the 11th European Conference on Computer Vision, (2010) September 5-11; Crete, Greece, pp. 213-226.

[21] B. Geng, D. Tao, and C. Xu, "DAML: Domain adaptation metric learning", IEEE Transactions on Image Processing, vol. 20, no. 10, (2011), pp. 2980-2989.

[22] J. Liu, Z. Sun, and X. Luo, "Review and Research Development on Domain Adaptation Learning", Acta Automatica Sinica, vol. 40, no. 8, (2014), pp. 1576-1600.

[23] J. Tao and S. Wang, "Kernel distribution consistency based local domain adaptation learning", Acta Automatica Sinica, vol. 39, no. 8, (2013), pp. 1295-1309.

[24] J. R. Matey, O. Naroditsky, K. Hanna, R. Kolczynski, D. J. Lolacono, S. Mangru, M. Tinker, T. M. Zappia, and W. Y. Zhao, "Iris on the move: Acquisition of images for iris recognition in less constrained environments", Proceedings of the IEEE, vol. 94, no. 11, (2006), pp. 1936-1947.

[25] K. Nguyen, C. Fookes, S. Sridharan, and S. Denman, "Quality-driven super-resolution for less constrained iris recognition at a distance and on the move", Information Forensics and Security, IEEE Transactions on, vol. 6, no. 4, (2011), pp. 1248-1258.

[26] Y. Lee, R. J. Micheals, J. J. Filliben, and P. J. Phillips, "Vasir: An open-source research platform for advanced iris recognition technologies", Journal of Research of the National Institute of Standards and Technology, vol. 118, (2013), pp. 218-259.

[27] L. Hoegaerts, J. A. Suykens, J. Vandewalle, and B. De Moor, "Subset based least squares subspace regression in RKHS", Neurocomputing, vol. 63, (2005), pp. 293-323.

[28] H. Proenca, S. Filipe, R. Santos, J. Oliveira, and L. A. Alexandre, "The ubiris. v2: A database of visible wavelength iris images captured on-the-move and at-a-distance", IEEE Transactions on Pattern Analysis and Machine Intelligence, vol. 32, no. 8, (2010), pp. 1529-1535.

[29] H. Hofbauer, F. Alonso-Fernandez, P. Wild, J. Bigun, and A. Uhl, "A Ground Truth for Iris Segmentation", the 22nd International Conference on Pattern Recognition, (2014) August 24-28; Stockholm, Sweden, pp. 527-532.

[30] C. Rathgeb, A. Uhl, and P. Wild, "State-of-the-Art in Iris Biometrics", in Iris Biometrics, Springer New York, New York, USA, (2013), pp. 21-36. 